\title{
IDENTIFICAÇ̃̃O DE FORMAS PARASITÁRIAS EM VEÍCULOS DE TRANSPORTE PÚBLICO DA CIDADE DE SÃO MATEUS, ESPÍRITO SANTO, BRASIL
}

\section{IDENTIFICATION OF PARASITIC FORMS IN PUBLIC TRANSPORT VEHICLES IN THE CITY OF SÃO MATEUS, ESPÍRITO SANTO, BRAZIL}

\author{
Jamille Santos Miossi ${ }^{1}$, Rafaela Souza Barros ${ }^{2}$, \& Marco Antônio Andrade de Souza ${ }^{3^{*}}$ \\ 123 Departamento de Ciências da Saúde do Centro Universitário Norte do Espírito Santo da Universidade \\ Federal do Espírito Santo, Rodovia BR 101 Norte, Km. 60, Bairro Litorâneo, CEP 29932-540, São Mateus. \\ 1 jamillimiossi@gmail.com² rafasouzabarros123@gmail.com 3* marco.souza@ufes.br
}

\section{ARTIGO INFO.}

Recebido em: 11.01.2021

Aprovado em: 02.03.2021

Disponibilizado em: 08.03.2021

\section{Palavras-chave:}

Parasitologia; Ônibus; Contaminação; Fita adesiva; São Mateus.

\section{KEYWORDS:}

Parasitology; Bus; Contamination; Scotch tape; São Mateus.

\section{*Autor Correspondente: Souza, M. A. A. de \\ RESUMO}

Introdução. Uma das grandes preocupações dos órgãos de saúde pública é a incidência de parasitoses, principalmente em países em desenvolvimento, como o Brasil. Essas doenças estão associadas com diversos fatores como deficiência de conhecimentos da população no que diz respeito à educação sanitária, destino incorreto de lixos, falta de saneamento básico e inúmeros processos de contaminação ambiental. Objetivo. Avaliar a contaminação por formas de enteroparasitos em rotas de ônibus na cidade de São Mateus. Material e Métodos. O método de escolha foi o de Graham, com modificações, que consiste na aposição de fita adesiva em suportes de apoio táteis, catracas, entradas e saídas dos ônibus, todas em alturas médias estimadas ao alcance das mãos, e posterior deposição da fita em lâminas de vidro por contato direto. O material obtido foi analisado no Laboratório de Parasitologia e Hematologia Clínica da Universidade Federal do Espírito Santo, campus São Mateus, em microscópio de luz (10X e 40X), sem qualquer adição de corantes. Resultados. Foram analisadas setenta e duas amostras, distribuídas entre as oito linhas de transporte público selecionadas no estudo. Os resultados indicaram a contaminação de
$50 \%$ dos veículos analisados e os principais parasitos e comensais encontrados foram Balantidium coli, Iodamoeba butschlii e Entamoeba coli. Conclusões. Os ônibus de transportes públicos da cidade de São Mateus apresentam potencial para a contaminação humana e podem gerar riscos para a população que utiliza este serviço.

\section{ABSTRACT}

Introduction. One of the major concerns of public health agencies is the incidence of parasitic diseases, especially in developing countries, such as Brazil. These diseases are associated with several factors such as the lack of knowledge of the population regarding health education, the incorrect destination of waste, lack of basic sanitation and numerous processes of environmental contamination. Objective. To evaluate the contamination by forms of enteroparasites in bus routes in the city of São Mateus. Methodology. The method used in this study was that of Graham, with modifications, that consists in the apposition of tape in tactile supports, turnstiles, entrances and exits of the buses, all in average estimated heights at the reach of the hands and later deposition in glass slides by direct contact. The obtained material was analyzed in the Laboratorio de Parasitologia e Hematologia Clínica of the Universidade Federal do Espírito Santo, campus São Mateus under light microscope (10X and 40X), without any addition of dyes. Results. A total of seventy-two samples were analyzed, distributed among the eight public transport lines selected in the study. The results indicated the contamination of $50 \%$ of the vehicles analyzed and the main parasites and commensal were Balantidium coli, Iodamoeba butschlii and Entamoeba coli. Conclusions. Public transportation buses in the city of São Mateus have potential for human contamination and can createrisks for the population that uses this service. 


\section{INTRODUÇÃo}

A prevalência das enteroparasitoses varia de acordo com a região e com a população estudada, fatores estes que contribuem para a morbidade e mortalidade de milhares de indivíduos em todo o mundo, em grande parte nos países considerados emergentes (Reis \& Carneiro, 2007), como o Brasil, que apresenta graves problemas de saneamento básico, enfrentados por grande parte da população, o que reflete em índices alarmantes de parasitoses intestinais (Reis \& Carneiro, 2007).

Dentre as parasitoses intestinais, aquelas cujos ciclos de transmissão ocorrem pelo contato com as formas infectantes presentes no solo contaminado, a prevalência da doença na população mundial varia de $20 \%$ e $50 \%$ (Who, 2021).

A questão de contaminação do homem por organismos eucariontes, como os protozoários e os helmintos, leva a consequências patológicas como má-absorção de nutrientes, diarreia e anemia, reduzindo sua capacidade de trabalho. Em crianças, por exemplo, pode ocasionar baixo rendimento escolar e déficit no crescimento, podendo levar o indivíduo até mesmo ao óbito (Rodrigues, Nishi, Guimarães, 2006; Ludwig et al. 2017).

Um aspecto relevante no processo de contaminação parasitária está na relação entre o homem e os animais, que vem se tornando cada vez mais próxima, principalmente com os animais de estimação que possuem um papel importante na estrutura familiar e social. Todavia, essa interrelação não fica limitada apenas a uma situação de coabitação familiar. Esses animais estão presentes em áreas públicas e, frequentemente, acabam depositando seus dejetos nesses espaços. Consequentemente, dejetos de animais parasitados no ambiente acabam provocando doenças em seres humanos (Lima et al. 2008).

Os ciclos evolutivos da maioria dos parasitos, tais como Giardia lamblia, Entamoeba histolytica, Ascaris lumbricoides e Taenia solium apresentam um padrão comum, uma vez que ocorrem no meio externo, cujas fezes humanas contaminadas com formas infectantes, provocam a contaminação do solo e a disseminação parasitária no ambiente (Mentz et al. 2004; Neves et al. 2016). Essas ocorrências afetam diferentes espaços urbanos, locais em que as pessoas caminham, assentam e mantêm contato, utilizando seus membros inferiores e também o apoio das mãos.

Nesse sentido, os ônibus de transporte coletivo representam um desses espaços, já que apresentam, diariamente, um grande fluxo de pessoas, tornando-se fonte para disseminação de microrganismos como bactérias, fungos e parasitos sobre seus usuários (Rodrigues, Nishi, Guimarães, 2006; Fernandes et al. 2012).

Assim, o presente estudo buscou detectar e avaliar a presença de formas parasitárias, nesse meio de locomoção, que podem afetar a saúde da população e contribuir para o fortalecimento da tomada de medidas de prevenção e da promoção da saúde.

\section{(ब)}




\section{Metodologia}

\subsection{LOCAIS DE ESTUDO}

A pesquisa foi realizada na cidade de São Mateus, Espírito Santo, entre março e julho de 2019. O município está localizado na Mesorregião Litoral Norte Espírito-Santense, a $64 \mathrm{Km}$ da divisa com o estado da Bahia e distante cerca de $220 \mathrm{~km}$ da capital Vitória (IBGE, 2019).

Foram avaliados os ônibus de transportes coletivos que fazem as seguintes rotas: Aroeira, Aviação, Cohab, Morada do Lago, Morada de Ribeirão, Roberto Silvares, Santo Antônio e Villages. Como critério para a escolha das rotas buscou-se avaliar aquelas com maior fluxo diário de usuários e abrangência de integração na cidade.

Para coleta das amostras foram verificadas catracas, saídas, entradas e barras de apoio paralelas ao corredor, dos ônibus, todas em alturas médias estimadas de alcance das mãos dos usuários.

Foram analisadas setenta e duas amostras, distribuídas entre as oito linhas de transporte coletivo mencionadas, resultando em nove amostras por linha.

\subsection{MATERIAIS}

Os materiais utilizados para os procedimentos de coleta e análise foram compostos por fita adesiva comum, lâminas de vidro, caixa de isopor e microscópio de luz.

\subsection{Metodologia de Coletas de Amostras}

Utilizou-se, para as coletas das amostras, a técnica desenvolvida por Graham no ano de 1941, específica para o diagnóstico de Enterobius vermicularis, com modificações. O método consiste em retirar um pedaço de 8 a $10 \mathrm{~cm}$ de fita adesiva transparente, aderindo-a nos locais que apresentam maior probabilidade de contato das mãos dos usuários do transporte coletivo, como por exemplo nos assentos, saídas, barras de apoio laterais e superiores dos veículos analisados. Posteriormente, a fita adesiva foi fixada na lâmina de vidro, previamente identificada.

As lâminas obtidas foram transportadas ao Laboratório de Parasitologia e Hematologia Clínica da Universidade Federal do Espírito Santo, campus São Mateus, acondicionadas em um recipiente térmico de isopor, e armazenadas em um refrigerador.

As análises das lâminas foram realizadas utilizando-se microscópio de luz nos aumentos de 10X e 40X, não sendo utilizado nenhum tipo de corante.

\section{RESUltados}

Nas pesquisas de formas parasitárias foram analisadas 72 amostras, observando-se positividade em quatro (5,6\%) delas. Balantidium coli $(1 / 1,4 \%)$, Iodamoeba butschlii $(1 / 1,4 \%)$ e Entamoeba coli $(3 / 4,2 \%)$, foram os parasitos e comensais encontrados.

Com relação às rotas dos ônibus, observou-se que a maior ocorrência por formas parasitárias se deu na rota Roberto Silvares, com destino a área hospitalar (Figura 1). 
Figura 1. Porcentagem da ocorrência de formas parasitárias de acordo com as rotas de transporte coletivo da cidade de São Mateus, Espírito Santo, Brasil.

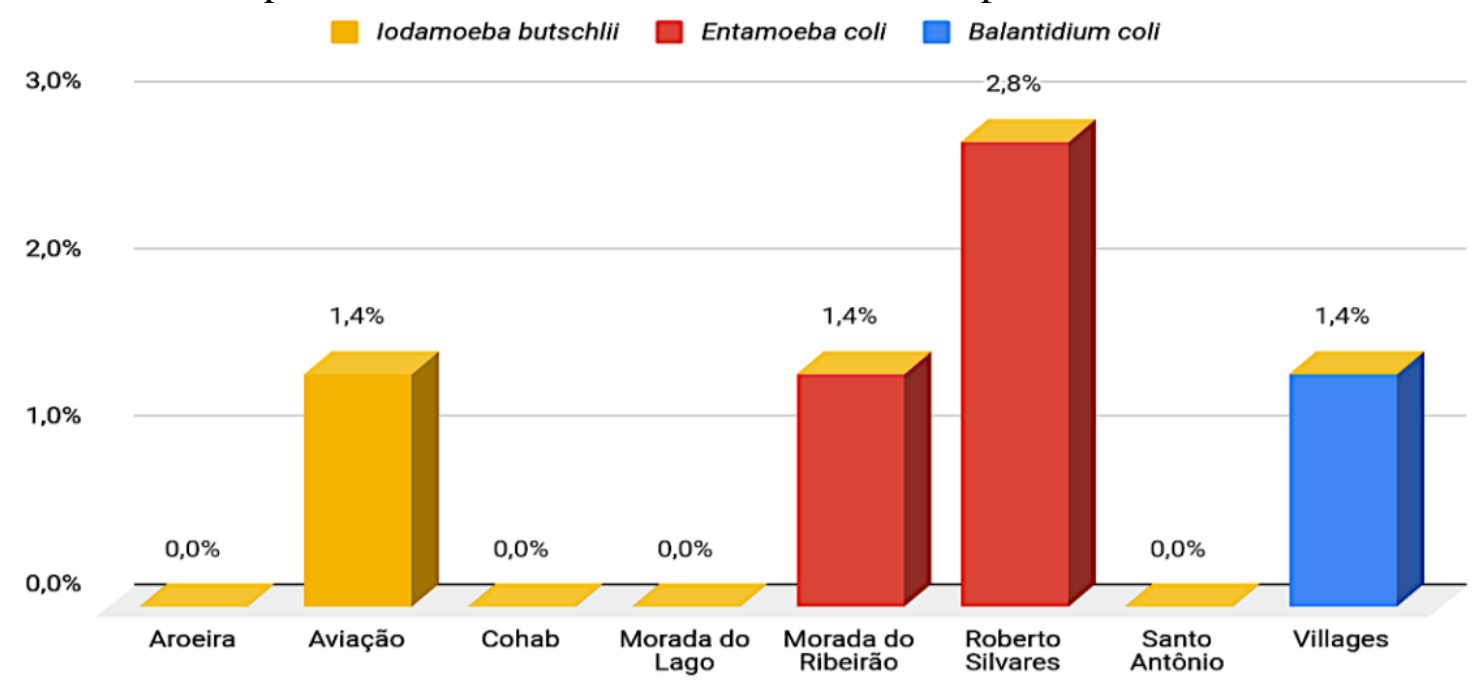

Por outro lado, as formas parasitárias foram encontradas com maior frequência nos horários de 17h (na linha Roberto Silvares), às 18h (na linha Villages), às 18h40min (na linha Aviação) e às 19h30min (na linha Morada do Lago).

Quanto ao local de encontro das formas parasitárias e comensais nos ônibus, observou-se que o cisto de $B$. coli foi encontrado em uma barra de apoio lateral, o cisto de $I$. butschlii em uma barra de apoio superior e os de $E$. coli foram encontrados em uma barra superior (Tabela 1). Os corrimões laterais e superiores tiveram índices de parasitos encontrados de 5,6\%, ao passo que os assentos tiveram incidência de 1,4\%. Nas saídas dos ônibus não foram verificadas ocorrências de formas parasitárias.

Tabela 1. Positividade para formas parasitárias de acordo com os locais de coleta das amostras no interior dos ônibus de transporte coletivo do município de São Mateus, Espírito Santo, Brasil.

\begin{tabular}{cccc}
\hline Locais de & \multicolumn{3}{c}{ Parasitos Intestinais } \\
amostragem nos ônibus & 1 & Entamoeba coli & Iodamoeba butschlii \\
\cline { 2 - 4 } & Balantidium coli & 2 & 1 \\
\cline { 2 - 4 } Barras Laterais/Superiores & 0 & 0 & 0 \\
Saídas & 0 & 1 & 0 \\
Bancos & 0 & & \\
\hline
\end{tabular}

\section{DISCUSSÃo}

Estima-se que cerca de 800 milhões de indivíduos, especialmente crianças, estão contaminados por parasitos intestinais. Dentre os mais prevalentes, no ano de 2020, destacam-se $A$. lumbricoides, T. trichiura, Ancilostomídeos, G. duodenalis e E. histolyticalE. dispar (Who, 2020) parasitos, esses, de fácil transmissão e cujas formas parasitárias podem resistir por meses no meio externo, como observado em diversos estudos sobre superfícies de objetos como fontes de transmissão de parasitos (Murta \& Massara, 2009; Damazio et al. 2015; Scholte et al. 2015).

Para a realização da pesquisa, em São Mateus, foram utilizadas técnicas parasitológicas laboratoriais semelhantes às descritas por Gomes e colaboradores (2016) que analisaram 250

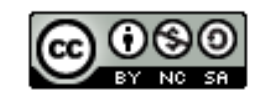


amostras na cidade de Patos de Minas, MG, provindas de transporte público e verificaram contaminação por estruturas parasitárias em $1,33 \%$ das amostras, resultado inferior ao encontrado em São Mateus, ao passo que Mendonça et al. (2008) ao estudarem a ocorrência de enteroparasitos na cidade de São Paulo obtiveram 4,17\% de positividade nas 120 amostras analisadas. Já Murta e Massara (2009) em análises na cidade Belo Horizonte, MG, observaram $17,3 \%$ de amostras positivas o que reforça a importância e necessidade de atenção e estudos contínuos relacionados às parasitoses intestinais e seus mecanismos de transmissão. Por outro lado, os maiores índices de contaminação de amostras coletadas em veículos de transportes públicos podem ser observados em trabalhos como os descritos por Fernandes et al. (2012) na cidade de Vitória, ES, e Andrade et al. (2019), em Diamantina, MG, que encontraram taxas de $70 \%$ e $78,24 \%$, respectivamente.

A presença de formas parasitárias em barras laterais e superiores e em assentos de veículos de transporte público na cidade de São Mateus indica potenciais focos de contaminação humana e este problema, por si só, não está somente relacionado com o transporte público, mas também à falta de higiene, ao desconhecimento da população sobre as doenças, à má qualidade de vida e, especialmente, ao saneamento básico precário que grande parte da população enfrenta no seu dia a dia.

Do ponto de vista higiênico-sanitário tais resultados refletem de forma significativa as práticas inadequadas de lavagem das mãos. Nesse sentido, é importante salientar a necessidade de maiores estudos que abordam a contaminação cruzada entre superfícies de objetos e indivíduos, a fim de garantir a segurança dos usuários de transportes coletivos. Contudo, é necessária uma mudança de comportamento da população de forma a reduzir a propagação de doenças causadas por enteroparasitos, assegurando uma melhor qualidade de vida.

\section{CONCLUSÃo}

Os ônibus de transporte coletivo da cidade de São Mateus apresentam potencial para a contaminação humana, constatado em formas parasitárias encontradas em algumas linhas urbanas.

O estudo realizado é de importante relevância para o fornecimento de informações epidemiológicas necessárias para promover medidas de intervenção, sendo fundamental a conscientização dos usuários de transporte coletivo para que este tipo de contaminação possa ser minimizado.

\section{REFERÊNCIAS BIBLIOGRÁFICAS}

Andrade, E. C., Leite, I. C. G., Rodrigues, V. De O., \& Cesca, M. G. (2010). Parasitoses intestinais: uma revisão sobre seus aspectos sociais, epidemiológicos, clínicos e terapêuticos. Revista APS, 13(2), 231-240.

Amorim, R. F., \& Souza M. A. A. (2016). Identificação de enteroparasitos na areia da praia de Guriri, São Mateus, ES. Scientia Vitae, 3(12): 1-9.

Andrade, S. S., Teodoro L. M., Viana, D. J. S., Sales, A. M. C., Oliveira, G. H. B., Bôas, S. V., \& Barata, R. A. (2019). Intestinal parasites in public transport buses from the city of Diamantina, Minas Gerais, Brazil. Research and Reports in Tropical Medicine, (8): 59-63.

Damazio, S. M., Maciel, J. S., Amorim, R. F., SOUZA, M. A. A. (2015). Occurrence of 
intestinal parasites in paper money circulating in the local trade of the city of São Mateus, Espírito Santo, Brazil. Hygeia: Revista Brasileira de Geografia Médica e da Saúde, 11, 12-19.

Fernandes, A. A. L., Rangel, C. D., Sena, C. J. C., Rangel, C. V., \& Moraes, R. (2012). Diversidade de bactérias, fungos e formas de resistência de parasitos em duas rotas de ônibus do transporte coletivo da grande Vitória-ES. Sapientia, 11(11): 39-45, 2012.

Gomes, T. M., Silva, J. O. R., \& Cardoso, S. R. A. (2016). Pesquisa de enteroparasitas em meios de transporte público urbano da cidade de Patos de Minas Gerais - Brasil. Psicologia e Saúde em Debate, 2(1): 74-99.

Graham, C. F. (1941). A device for the diagnosis of Enterobius infection. American Journal of Tropical Medicine, 21: 159-161.

IBGE. Instituto Brasileiro de Geografia e Estatística. Brasil em síntese: São Mateus. 2019. Recuperado de https://cidades.ibge.gov.br/brasil/es/sao-mateus/panorama.

Lima, A. M. A., Alves, L. C., Faustino, M. A. G., \& Lira, N. M. S. (2010). Percepção sobre o conhecimento e profilaxia das zoonoses e posse responsável em pais de alunos do pré-escolar de escolas situadas na comunidade localizada no bairro de Dois Irmãos na cidade do Recife (PE). Ciência \& Saúde Coletiva, 1(1): 1457- 1464.

Ludwig, K. M., \& Conte, A. O. C. (2017). Enteroparasitoses em crianças de uma creche na cidade de Assis/SP - antes e depois de campanhas educativas. Saúde, 43(2), 265-345.

Mendonça, R. G. M., Olival, G. S., Mimica, L. M. J., Navarini, A., Paschoalotti, M. A., \& Chieffi, P. P. (2008). Potencial infeccioso do transporte público de passageiros da cidade de São Paulo. Arquivos Médicos dos Hospitais e da Faculdade de Ciências Médicas da Santa Casa de São Paulo, 53(2): 53-5.

Mentz, B. M., Rott, B. M., Jacobsen, I. S., Baldo, G., Júnior, R. V. (2004). Frequência de ovos de Toxocara spp. em três parques públicos na cidade de Porto Alegre, Rio Grande do Sul, Brasil. Revista de Patologia Tropical, 33(1): 105-112.

Murta, F. L., \& Massara, C. L. (2009). Presença de ovos de helmintos intestinais em ônibus de transporte público em Belo Horizonte - Minas Gerais, Brasil. Revista de Patologia Tropical, 38(3): 207-212.

Neves, D. P., Melo, A. L., Linardi, P. M., \& Vitor, R. W. A. (2016). Parasitologia humana, 13ed., São Paulo: Atheneu, 616p.

Reis, R. M., \& Carneiro, L. C. (2007). Indicador higiênico-parasitário em manipuladores de alimentos em morrinhos, GO. Rev. Estudos de Biologia, 29(68/69): 313-317.

Rodrigues, A. P. C., Nishi, C. Y. M., \& Guimarães, A. T. B. (2006). Levantamento de bactérias, fungos e formas de resistência de parasitos em duas rotas de ônibus do transporte coletivo de Curitiba, Paraná. RUBS, 2(2): 24-31.

Scholte, R. G. C., Carvalho, O. S., Lima, G. M. N., Araújo, A. D., \& Massara, C. L. (2015). Pesquisa de Ovos de Helmintos Intestinais nos Terminais Aeroportuários Carlos Drummond de Andrade (Pampulha) - Belo Horizonte e Tancredo Neves - Confins - Minas Gerais. Revista de Patologia Tropical, 44(3): 303-311.

Who. World Health Organization. Intestinal worms. 2020. Recuperado de https://www.who.int/intestinal_worms/more/en/. 